\title{
Effects of MYBPC3 loss-of-function mutations preceding hypertrophic cardiomyopathy
}

Adam S. Helms, ${ }^{1}$ Vi T. Tang, ${ }^{1}$ Thomas S. O'Leary, ${ }^{2}$ Sabrina Friedline, ${ }^{1}$ Mick Wauchope, ${ }^{1}$ Akul Arora, Aaron H. Wasserman, ${ }^{3}$ Eric D. Smith, ${ }^{1}$ Lap Man Lee, ${ }^{4}$ Xiaoquan W. Wen, ${ }^{5}$ Jordan A. Shavit, ${ }^{6}$ Allen P. Liu, ${ }^{4,7}$ Michael J. Previs, ${ }^{2}$ and Sharlene M. Day,3

'Department of Internal Medicine, Division of Cardiovascular Medicine, University of Michigan, Ann Arbor, Michigan, USA 2Department of Molecular Physiology \& Biophysics, University of Vermont, Burlington, Vermont, USA. ${ }^{3}$ Departments of Molecular and Integrative Physiology, ${ }^{4}$ Mechanical Engineering, ${ }^{5}$ Biostatistics, ${ }^{6}$ Pediatrics, and ${ }^{7}$ Biophysics, University of Michigan, Ann Arbor, Michigan, USA.

Mutations in cardiac myosin binding protein C (MyBP-C, encoded by MYBPC3) are the most common cause of hypertrophic cardiomyopathy (HCM). Most MYBPC3 mutations result in premature termination codons (PTCs) that cause RNA degradation and a reduction of MyBP-C in HCM patient hearts. However, a reduction in MyBP-C has not been consistently observed in MYBPC3-mutant induced pluripotent stem cell cardiomyocytes (iPSCMs). To determine early MYBPC3 mutation effects, we used patient and genome-engineered iPSCMs. iPSCMs with frameshift mutations were compared with iPSCMs with $M Y B P C 3$ promoter and translational start site deletions, revealing that allelic loss of function is the primary inciting consequence of mutations causing PTCs. Despite a reduction in wild-type mRNA in all heterozygous iPSCMs, no reduction in MyBP-C protein was observed, indicating protein-level compensation through what we believe is a previously uncharacterized mechanism. Although homozygous mutant iPSCMs exhibited contractile dysregulation, heterozygous mutant iPSCMs had normal contractile function in the context of compensated MyBP-C levels. Agnostic RNA-Seq analysis revealed differential expression in genes involved in protein folding as the only dysregulated gene set. To determine how MYBPC3-mutant iPSCMs achieve compensated MyBP-C levels, sarcomeric protein synthesis and degradation were measured with stable isotope labeling. Heterozygous mutant iPSCMs showed reduced MyBP-C synthesis rates but a slower rate of MyBP-C degradation. These findings indicate that cardiomyocytes have an innate capacity to attain normal MyBP-C stoichiometry despite $M Y B P C 3$ allelic loss of function due to truncating mutations. Modulating MyBP-C degradation to maintain MyBP-C protein levels may be a novel treatment approach upstream of contractile dysfunction for HCM.

Conflict of interest: The authors have declared that no conflict of interest exists.

Copyright: (ㄷ) 2020, American Society for Clinical Investigation.

Submitted: September 25, 2019 Accepted: December 10, 2019 Published: January 30, 2020.

Reference information: /CI Insight. 2020;5(2):e133782.

https://doi.org/10.1172/jici. insight.133782.

\section{Introduction}

Mutations in cardiac myosin binding protein $\mathrm{C}$ (MyBP-C, encoded by $M Y B P C 3)$ are the most common cause of familial hypertrophic cardiomyopathy (HCM), an autosomal dominant inherited cardiomyopathy with incomplete penetrance (1). Cardiac MyBP-C is a flexible, rod-like protein (Figure 1A) that is a key component of the cardiac sarcomere, the heart's most basic contractile unit. Within the sarcomere, MyBP-C's C-terminal C10 domain (Figure 1A) is strongly bound to the myosin filament backbone, and its N-terminal domains bind to either the myosin head or actin filament to modulate calcium-activated actomyosin sliding, which powers cardiac muscle contractility $(2,3)$. While heterozygous mutations in $M Y B P C 3$ are the most common cause of hereditary HCM, homozygous MYBPC3 frameshift mutations lead to a severe form of HCM that is lethal in early infancy in humans, underscoring MyBP-C's importance in normal cardiac function $(4,5)$.

Among patients with HCM, 90\% of MYBPC3 mutations are heterozygous frameshift, nonsense, or splice site mutations that result in premature termination codons (PTCs) on 1 allele (1). As such, these mutations are thought to result in HCM from an allelic loss of function via NMD of PTC-containing transcripts (6), leading to a reduction in MyBP-C content in the sarcomere. Alternatively, these mutations may result in production 


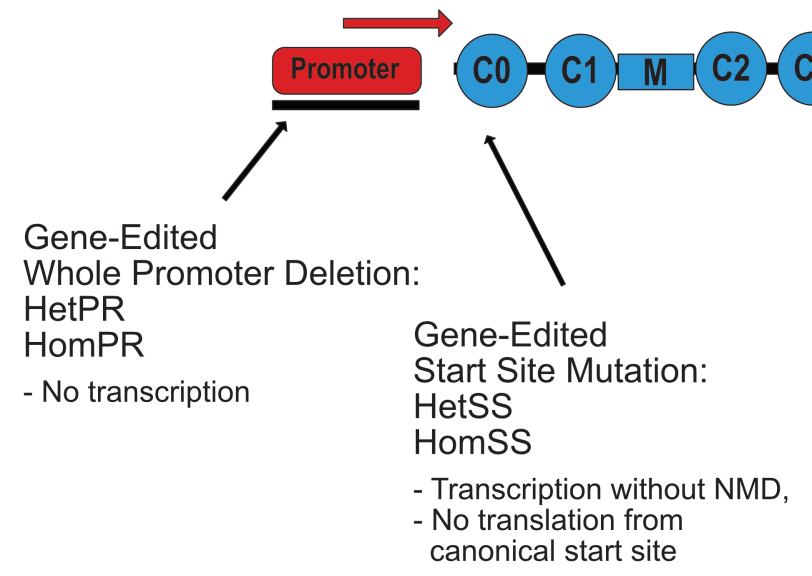

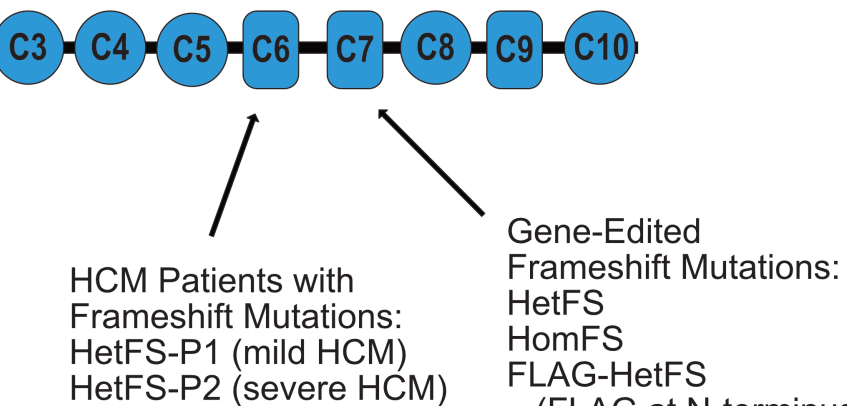

(FLAG at N-terminus)

- Transcription with NMD

- Transcription with NMD

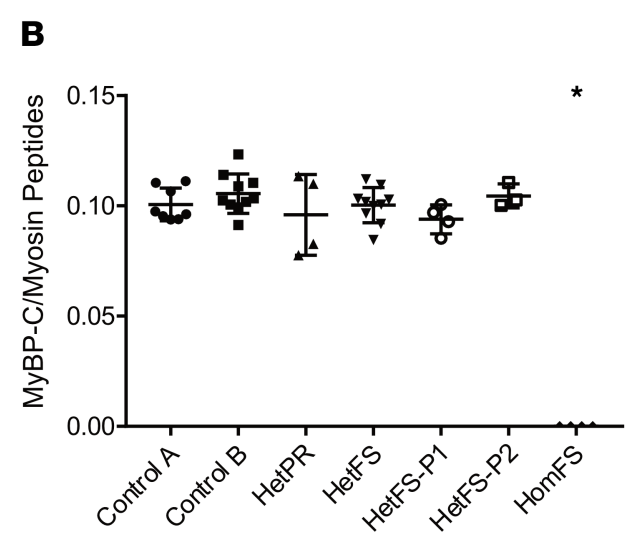

\section{C}

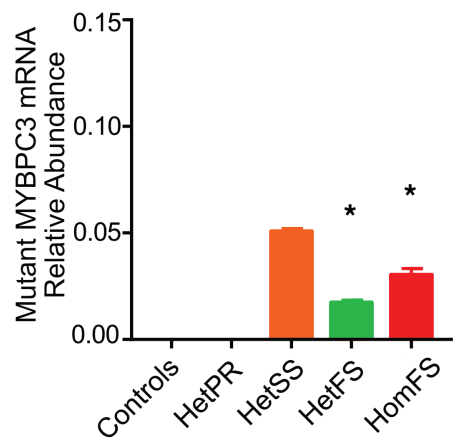

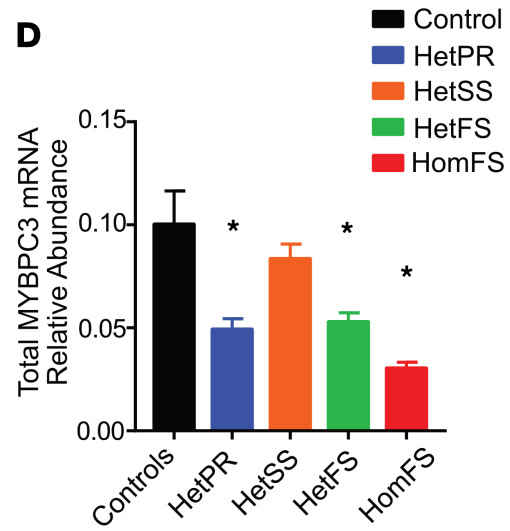

Figure 1. MYBPC3-mutant iPSCMs exhibit compensated MyBP-C protein levels despite reduced mRNA. (A) Diagram of MyBP-C depicts the overall strategy for determining МУВРС3 mutation mechanisms through generation of an allelic series of mutations in the same genetic background, in addition to patient iPSC lines. (B) MyBP-C protein levels were determined from spectral counts by mass spectroscopy analysis and normalized to myosin abundance. MyBP-C levels were equivalent among the gene-edited heterozygous, HCM patient, and control lines, but MyBP-C was undetectable in the homozygous frameshift line (1-way ANOVA used for statistical comparison against controls; $\left.{ }^{*} P<0.05\right)$. (C) The mutant $M Y B P C 3$ mRNA relative abundance was determined by using the proportion of RNA-Seq read counts from the mutant allele, normalized to total control expression level. For HetPR, no mutant mRNA is expressed from the mutant allele, while HetSS has approximately 50:50 mutant to wild-type allele because the mutant mRNA for this line is not susceptible to NMD. In contrast, the HetFS and HomFS lines have a reduction in mutant mRNA because of NMD. The relative mutant proportion in HetCT and HomCT was compared with HetSS using 1-way ANOVA with $P<0.05$ as significant (see Methods). The relative mutant proportion in HetFS and HomFS was compared with HetSS using 1-way ANOVA with $P<0.05$ as significant (see Methods). (D) Total MYBC3 mRNA abundance was determined using RNA-Seq for robust normalization with a DESeq2 (55) and remove unwanted variation-sequencing RUV-Seq (56) pipeline (see Methods). Relative abundance was determined using dispersion estimates for each gene fit to a binomial generalized linear model with DESeq2, which yields a log, fold change estimate and SEM for each gene, converted here to relative abundance on a nonlogarithmic scale. The Wald test was used for statistical significance testing with multiple-testing correction for 16,819 genes tested (adjusted $P<0.05$ was considered significant). iPSC, induced pluripotent stem cell; iPSCM, induced pluripotent stem cell cardiomyocyte; NMD, nonsense-mediated RNA decay.

of truncated MyBP-C, though truncated MyBP-C has never been detected in human heart tissue (6-8). Data from adult human HCM hearts collected during surgical myectomy demonstrate that MyBP-C content within the sarcomere is reduced by approximately 40\% during this advanced phase of HCM (9). These findings support the concept of MyBP-C haploinsufficiency in HCM, seeing as an allelic loss of function is sufficient to result in a pathogenic reduction in MyBP-C content. However, this reduction in MyBP-C content has been only variably observed in stem cell models of HCM harboring MYBPC3 mutations (10-13). We hypothesized that this could be due to temporal changes in MyBP-C expression during the development of HCM.

Sarcomere proteins, including MyBP-C, are continually renewed through protein synthesis and degradation (14), thus raising the possibility that MyBP-C levels may be modulated during development of, maturation of, or periods of stress in cardiomyocytes. Supporting this concept, mouse models with heterozygous $M Y B P C 3$ mutations only demonstrate reductions in MyBP-C content in response to transaortic constriction (15). Such modulation of MyBP-C levels could be due to transcriptional upregulation of the 
nonmutant allele to produce normal amounts of wild-type MyBP-C and/or slowing of the normal rate of MyBP-C degradation, to increase the half-life of MyBP-C within the sarcomere. This capacity may only be diminished once patients exhibit an overt hypertrophic phenotype. However, access to heart tissue from humans with MYBPC3 mutations before the development of HCM is not feasible.

To probe the relationship between $M Y B P C 3$ allelic loss-of-function mutations, protein production, and contractile function in cardiac muscle cells prior to the development of HCM, we leveraged iPSC models and a combination of fluorescence cellular imaging, quantitative mass spectrometry, single-cell biophysical techniques, and RNA-Seq. To fully define allelic loss-of-function effects, we used a spectrum of MYBPC3-mutant iPSC lines, including patient-derived iPSCs and genome-engineered iPSCs with both heterozygous and homozygous allelic loss-of-function mutations by different mechanisms. We find that heterozygous MYBPC3-mutant iPSCMs, despite reduced mRNA levels, have compensated, normal MyBP-C protein abundance, which preserves contractile function in the heterozygous mutant iPSCMs. Through mass spectroscopy of isotopically labeled samples, we identify a critical mechanism for this compensation to be a reduction in the rate of MyBP-C degradation. Collectively, these findings support a concept that intervention during early HCM development to maintain MyBP-C equilibrium could mitigate disease pathogenesis before contractile dysfunction occurs.

\section{Results}

Patient-derived and genome-engineered MYBPC3-mutant iPSC models. To determine the immediate cellular consequences of heterozygous PTC-causing $M Y B P C 3$ mutations, including potential compensatory mechanisms to regulate MyBP-C levels, we obtained patient-derived iPSC lines with frameshift mutations, and we genome engineered frameshift mutations into an established control iPSC line to generate otherwise isogenic lines (Figure 1A, Supplemental Figure 1, and Supplemental Table 1; supplemental material available online with this article; https://doi.org/10.1172/jci.insight.133782DS1). We used 2 previously published patient iPSC lines - 1 line was from a patient with severe, pediatric-onset HCM, and the other was from this patient's parent, who had only mild HCM, as previously described $(13,16)$. In the genome-edited lines, frameshift mutations were introduced at a similar location as the patient lines (Figure 1A), although PTC-causing mutations in HCM occur throughout the gene, indicating that the precise location of the frameshift is likely unimportant (1). Both heterozygous and homozygous clones were selected, with the homozygous line modeling the extreme end of the spectrum of normal MyBP-C loss, as in severe infantile cardiomyopathy (Figure 1A) (4, 5). Both patient and gene-edited frameshift mutant lines were expected to result in mutant MYBPC3 mRNA susceptible to NMD.

To verify the presence or absence of truncated MyBP-C in the context of a heterozygous, PTC-causing MYBPC3 mutation, we also performed a second round of gene editing on the engineered heterozygous frameshift mutant line, to create a separate line containing an N-terminal FLAG epitope tag on the mutant allele (Figure 1A). The FLAG frameshift line was used only to test for the presence of truncated protein, while all functional characterization was performed in the original heterozygous frameshift mutation line.

Next, to model allelic loss of function without susceptibility to NMD, we engineered whole promoter deletion lines. Because the $M Y B P C 3$ promoter has not previously been reported, we determined the DNAse-sensitive 5' region from UCSC Genome Browser and used simultaneous 5' and 3' guide (gRNAs) to remove the entire 185-bp region (Supplemental Figure 2, A and B). Although we selected a heterozygous clone for detailed study, we also selected a homozygous promoter deletion iPSC line to validate complete ablation of $M Y B P C 3$ expression. This latter line differentiated similarly to controls but totally lacked MyBP-C expression by immunofluorescence, confirming complete knockout (Supplemental Figure 2C).

Finally, we engineered a translational start site mutation line (disrupting the ATG) to model allelic loss of function with intact mRNA but without a PTC to trigger NMD (Figure 1A and Supplemental Figure 3). As described below, this line unexpectedly exhibited translational reinitiation with weak expression from a downstream alternative start site (Supplemental Figure 3). Therefore, this line was primarily used to examine effects on mRNA regulation, while the heterozygous frameshift, homozygous frameshift, and heterozygous promoter deletion lines were additionally used for detailed cellular and functional assays.

Taken together, if allelic loss of function is the primary pathogenic mechanism in MYBPC3-mutant HCM, then the above heterozygous gene-edited mutant MYBPC3 iPSC lines would all be expected to have indistinguishable cellular phenotypes given their isogenic background.

Wild-type MyBP-C protein level is compensated in iPSCMs with heterozygous allelic loss-of-function mutations but absent with homozygous loss-of-function mutations. We previously reported that heart tissue from patients with tissue-level myocardial hypertrophy and MYBPC3 frameshift or splice mutations exhibit an approximately 
$40 \%$ reduction in MyBP-C protein. However, we also previously demonstrated no reduction of MyBP-C in an embryonic stem cell model with a frameshift mutation, similar to a recent report from an iPSC model $(10,11)$. Therefore, we first quantified MyBP-C levels in patient and genome-engineered iPSCMs at 25 days after differentiation using mass spectroscopy to establish whether protein-level compensation is attained in the present models. Myosin was selected for normalization because it is the most abundant protein in the sarcomere and has a molecular weight within an order of magnitude of MyBP-C - these characteristics enable accurate determination of the relative molar abundance of the proteins (9). We found no difference in MyBP-C level normalized to myosin (Figure 1B), indicating that as-yet-uncharacterized mechanisms restore the relative MyBP-C abundance in all the heterozygous models. In contrast, the homozygous frameshift mutation line exhibited an absence of MyBP-C by mass spectroscopy (within an estimated 0.5\% sensitivity of the method), indicating that this iPSCM line could be used as a model of severe MyBP-C loss for the present studies. Although peptides shared in common between $\alpha$ - and $\beta$-myosin were used for quantification, we also observed no significant difference in $\beta$-myosin proportion in differentiated heterozygous $(59 \% \pm 20 \%)$ or homozygous $(65 \% \pm 13 \%)$ iPSCMs versus control iPSCMs $(66 \% \pm 17 \%, P=0.27)$.

Heterozygous allelic loss-of-function mutation types lead to a non-compensated reduction in wild-type MYBPC3 $m R N A$ transcription. Having demonstrated equivalent wild-type MyBP-C protein levels among all the heterozygous $M Y B P C 3$-mutant lines, we next quantified mutant and wild-type $M Y B P C 3$ mRNA levels for each of the isogenic heterozygous models. Frameshift $M Y B P C 3$ mutations that introduce PTCs render the resultant mRNA transcripts susceptible to NMD, and we previously showed that NMD removes on average $68 \%$ of mutant $M Y B P C 3$ transcripts in human heart tissue (6). In the isogenic heterozygous frameshift mutation model here, we found a similar level of degradation of the mutant mRNA (66\% $\pm 5 \%$ ), indicating that NMD is equivalently active in the early cellular model and hypertrophic heart tissue. To test whether there is a saturation of NMD capacity upon increasing mutant MYBPC3 mRNA burden, we compared the efficiency of NMD in heterozygous and homozygous frameshift mutant lines and found a similar rate of degradation $(66 \% \pm 5 \%$ vs. $70 \% \pm 6 \%, P=\mathrm{NS})$. This constant rate of degradation by NMD resulted in a residual $17 \%$ of mutant mRNA in the heterozygous frameshift line and $30 \%$ mutant mRNA in the homozygous line (i.e., proportionally similar), indicating neither an upregulation nor a saturation of NMD capacity (Figure 1C). We also quantified the mutant mRNA proportion in the heterozygous translational start site mutant line. As expected, mutant mRNA accounted for about half of total mRNA in this line, consistent with an expected lack of NMD of the mutant mRNA, because the mutation does not result in a PTC (Figure 1C).

Next, we compared the total MYBPC3 mRNA abundance among lines. We found that total MYBPC3 mRNA levels were similarly reduced by half in both the heterozygous frameshift (with NMD) and heterozygous promoter deletion (without NMD) iPSCMs, consistent with a complete lack of compensatory upregulation of mRNA level in either line (Figure 1D). The heterozygous start site mutation line exhibited a similar total mRNA abundance as controls, but because the $50 \%$ contribution from the mutant allele (as above) lacks the normal translational start site, only about half of the total mRNA is functional wild-type mRNA in this line (Figure 1D).

Taken together, these findings indicate that there is no innate compensation to maintain normal wild-type mRNA expression levels of $M Y B P C 3$ in the presence of a single-allele loss-of-function mutation, regardless of whether NMD is triggered. These findings also show that all the mutant alleles studied are loss of function from the standpoint of mRNA expression and that the compensation must occur posttranscriptionally (11).

Myofilament stoichiometry, myofibrillogenesis, and early stages of iPSCM growth are not affected by heterozygous or homozygous MYBPC3 mutations. Intracellular myofibrillar disarray is a defining feature of hypertrophic cellular remodeling in adult HCM heart tissue collected during septal myectomies (17). We first tested whether reductions in MYBPC3 transcripts or MyBP-C protein affect the generalized myofilament stoichiometry, which might consequently affect myofibrillogenesis, using RNA-Seq and mass spectroscopy. We found no disruption of other myofilament genes' transcript or protein abundance, even with MyBP-C ablation in the homozygous line (Figure 2, A and B). Next, to determine whether myofibrillar disarray occurs during early development, we characterized the effect of both heterozygous and homozygous MYBPC3 mutations on myofibrillar alignment and density in iPSCMs. We examined myofibrillar alignment 7 days following replating of iPSCMs onto micropatterned rectangles (25 days after differentiation), which avoided confounding by stochastic variation in cell shape $(18,19)$. The myofibrils were consistently aligned with the iPSCM long axes in all cells, including in the presence of severe MyBP-C loss in the homozygous line 
(Figure 2C; additional examples in Supplemental Figure 4). In addition, MyBP-C was consistently localized within 2 discrete bands near the center of each sarcomere in the tested heterozygous lines (Figure 2C).

Using the same assay, we tested whether evidence of hypertrophy is present in the MYBPC3-mutant models. Because hypertrophy in human HCM is caused by the addition of myofibrils in parallel, we measured the number of myofibrils across each cell, blinded to genotype. We found no difference in the numbers of myofibrils in control versus mutant iPSCMs, including the homozygous line (Figure 2, D-E, and Supplemental Figure 4). Taken together, these findings indicate that myofibrillogenesis between cell lines is independent of either heterozygous or homozygous MYBPC3 mutations and that the iPSCMs do not demonstrate an overt hypertrophic phenotype during the developmental period we studied.

Intercellular alignment of iPSCMs in micropatterned 2D tissues is not disrupted by MYBPC 3 mutations. In addition to intracellular myofibrillar disarray, intercellular disorganization is a key pathologic finding in remodeled HCM tissue, but whether this pathologic process begins during early cardiac development has not been clear (17). Earlier work from our group indicated that embryonic stem cell-derived iPSCs harboring $M Y B P C 3$ mutations may cause intercellular disorganization when comparing nonisogenic lines as anisotropic (nonaligned) 2D tissues, which may be subject to more stochastic variability (10). To determine the precise impact of $M Y B P C 3$ mutations on myofibrillar arrangement during tissue formation, we cultured purified iPSCMs from the genome-edited iPSC lines on row micropatterns to induce isotropic (aligned) growth of $2 \mathrm{D}$ monolayer cardiac tissues $(20,21)$. We assessed the capacity of iPSCMs from the tested lines to generate aligned, 2D tissues with $Z$ axis registration between adjacently coupled cardiomyocytes. We found that all tested MYBPC3-mutant iPSCMs demonstrated excellent myofibrillar alignment both within each cardiomyocyte and between adjacent cardiomyocytes, even with ablation of MyBP-C in the homozygous frameshift line (Figure 3). This demonstrated that, under our culture conditions, the genome-edited iPSCs harboring MYBPC3 mutations did not display intercellular disorganization when compared to isogenic control lines (10).

Truncated MyBP-C is undetectable in human iPSCS with heterozygous frameshift mutations in MYBPC3. The homozygous MYBPC3 frameshift mutation line had no detectable MyBP-C within the threshold of measurable signal-to-noise ratio ( $\sim 5 \%$ ) of control MyBP-C levels in the homozygous frameshift iPSCMs, consistent with efficient clearance of truncated protein from the residual 30\% mutant mRNA level (Figure 1, B and C). Consistent with this finding, no MyBP-C was apparent with immunofluorescent imaging of these cells when thresholding the fluorescence signal similar to controls and heterozygous lines (Figure 2C). With background subtraction and amplification of the fluorescence, a low-level signal was detectable with imprecise localization in myofibrils (Supplemental Figure 5D). Together with the mass spectroscopy data, this finding indicates a very low level of mutant MyBP-C expression in the homozygous frameshift line $(<0.5 \%)$.

Because $17 \%$ residual mutant mRNA was also present in the heterozygous frameshift gene-edited iPSC line, we additionally introduced a FLAG epitope tag in phase with the mutant allele in the heterozygous frameshift line, creating an additional line that was used to definitively determine whether low levels of mutant protein could be detected (Supplemental Figure 5, A-C). No mutant protein was detected by immunofluorescence or by immunoprecipitation using a FLAG antibody with high sensitivity and specificity (Supplemental Figure 5, E and F). Taken together, these data demonstrate that truncated mutant peptides, if expressed at all in cardiomyocytes with heterozygous $M Y B P C 3$ mutations, are rapidly degraded such that any scarce residual amount is unlikely to play a role in disease pathogenesis.

Presence of low abundance of mutant MyBP-C from an alternative start site with translational start site deletion. Although introduction of a translational start site deletion was anticipated to result in a nonfunctional mutant allele, unexpectedly, MyBP-C was detected both by Western blot and by immunofluorescence in the homozygous mutant line (Supplemental Figure 3, C and D). The Western blot band exhibited a weaker expression level relative to actin by Western blot and was present at a lower mass $(\sim 130 \mathrm{kDa})$ than wildtype MyBP-C (Supplemental Figure 3C), most consistent with weak expression from a noncanonical alternative start site (22). Mass spectroscopy was used to confirm the identity of this protein as MyBP-C lacking the initial N-terminal $\sim 102$ amino acids, corresponding with initiation from the next downstream ATG in exon 3 as an alternative start site (Supplemental Figure $3 \mathrm{E}$ ). The heterozygous start site mutation line also expressed the $130-\mathrm{kD}$ alternative start site MyBP-C but only at $7 \% \pm 2 \%$ of the full-length abundance (Supplemental Figure 3C). Mutations in MYBPC3 HCM are reported both at the canonical ATG and between the canonical ATG and the next downstream ATG, indicating that these models are potentially relevant to the human disease (1). Taken together, we demonstrate, possibly for the first time, mutant MyBP-C 
A

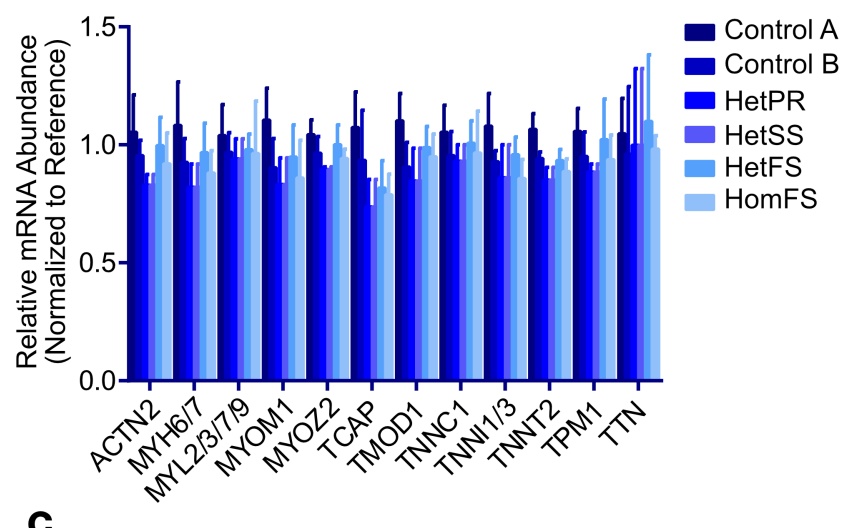

B

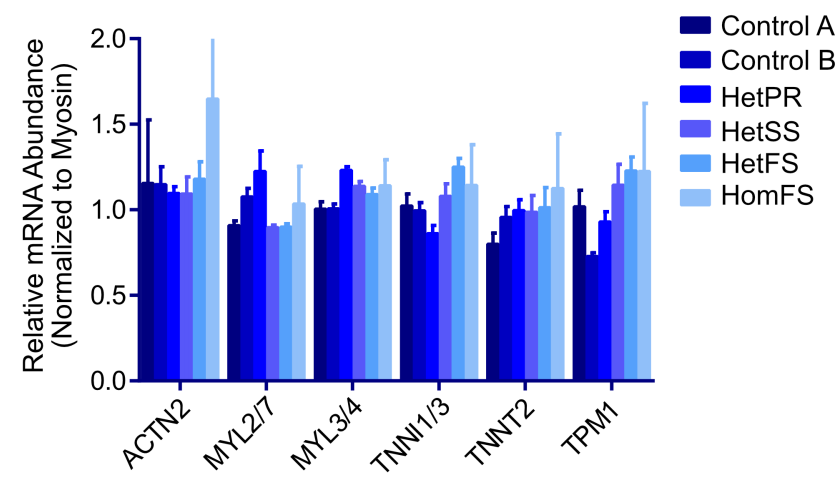

Intensity Plot
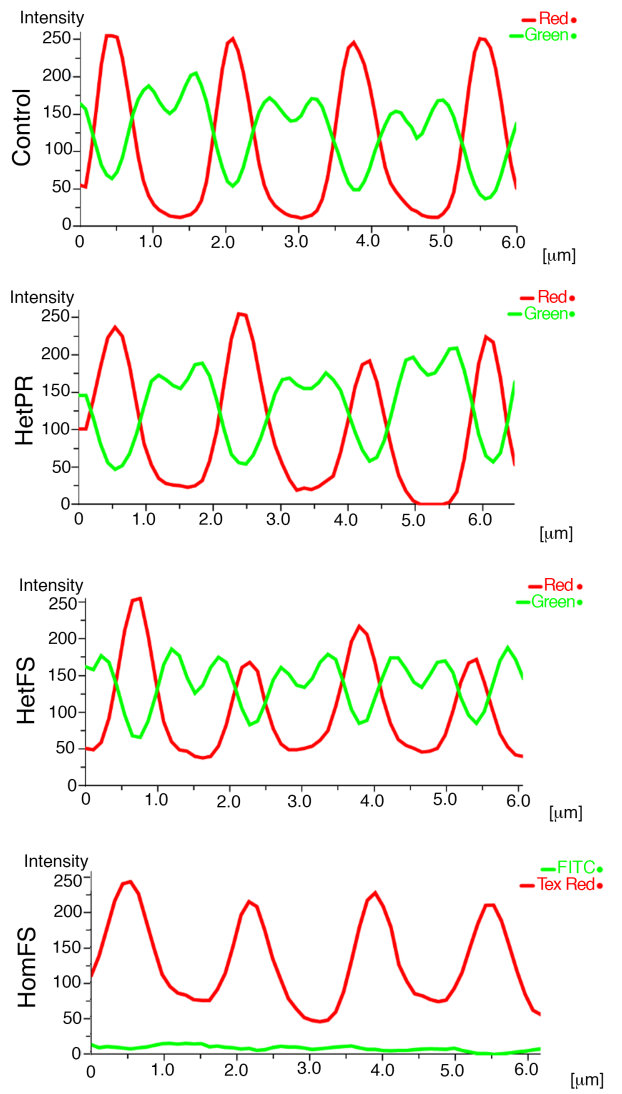

E

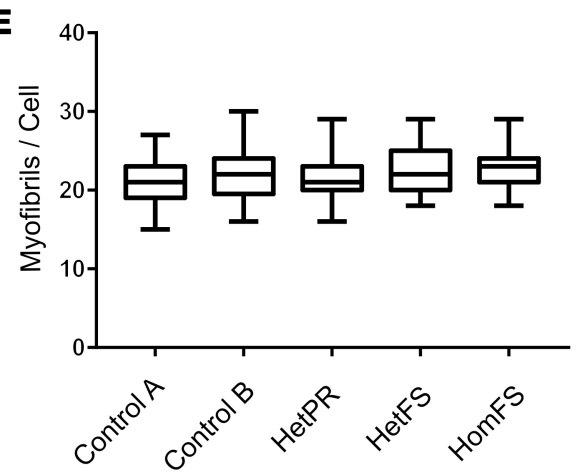


Figure 2. Myofilament stoichiometry, myofibrillogenesis, and early stages of iPSCM growth are not affected by heterozygous or homozygous MYBPC3 mutations. (A) Relative mRNA abundance of the major sarcomere genes (except MYBPC3) is not different among the isogenic MYBC3-mutant lines compared to controls, even in the case of the homozygous mutant line. Relative expression levels and statistical significance were determined from RNA-Seq analysis as in Figure 1D. Read counts for gene paralogs (e.g., MYH6 and MYH7) were coalesced for comparison because the objective was to determine overall stoichiometry of sarcomere gene expression and not effects on developmental state, which may be confounded by some variability in the iPSCM model system. (B) Mass spectroscopy quantification of sarcomere proteins (other than MyBP-C), normalized to total myosin, demonstrates no significant alteration in sarcomere protein levels. (C) Representative images from single 7:1 rectangular patterned iPSCMs demonstrate robust myofibrillar development and alignment among both mutant lines and control lines, including the homozygous mutant line. MyBP-C localization is normal in all heterozygous lines but is not observed when imaged with the same imaging parameters in the homozygous line (see also Supplemental Figure 5; MyBP-C detected with anti-CO-M domain antibody). Insets: $\times 2.5$ magnification. (D) Example of variability in myofibrillar density among control iPSCMs. This variability was observed among all control and mutant iPSCM lines and was used as an approach to quantify myofibrillar growth that would represent a cellular phenotype of cardiac hypertrophy. (E) To quantify myofibrillar hypertrophy, which is characterized by parallel addition of myofibrils, blinded myofibrillar counts on individual micropatterned iPSCMs were performed for each line ( $N>100$ cells per line) and showed no difference among isogenic lines, including the homozygous line (ANOVA, with $P<0.05$ as significant). The box plots depict the minimum and maximum values (whiskers), the upper and lower quartiles, and the median. The length of the box represents the interquartile range.

expressed from an alternative translational start site; however, ultimately these mutant alleles may still act primarily by loss of function because of low efficiency of the alternative start site.

Contractile function is preserved in iPSCMs with heterozygous MYBPC3 mutations, while severe MyBP-C loss enhances contractile deceleration. MyBP-C is a critical regulator of actin-myosin contractile dynamics, and reductions in MyBP-C content because of heterozygous mutations have been linked to hypercontractile function in adult human hearts at the molecular and cellular levels $(2,9)$. However, because we demonstrated that the heterozygous MYBPC3-mutant iPSCMs exhibit compensation of MyBP-C levels (Figure 1B), contractile function may not be perturbed in these cells, while the homozygous $M Y B P C 3$-mutant iPSCMs would be expected to exhibit effects on contractility from severe MyBP-C loss. To quantify contractile effects of these mutations, we measured contractile dynamics in single iPSCMs on micropatterned polyacrylamide gels by traction force microscopy (Figure 4A and Supplemental Figure 6) (18, $19,23,24)$. This method avoids confounding by effects of stochastic variability in cell shape that occur independent of genotype (19). In addition, we observed substantial variability in contractile force in control iPSCMs because of variability in myofibrillar density (Supplemental Figure 6, B and C). To obviate this technical issue, we used a live-cell actin-labeling method to select (blinded to genotype) only iPSCMs with robust myofibrillar development for analysis (see Methods). We identified no difference in maximum force, contractile kinetics, or relaxation kinetics with isogenic heterozygous frameshift or promoter deletion $M Y B P C 3$ mutations (Figure 4, C-F). In contrast, the homozygous frameshift mutation line demonstrated increased contractile velocity and decreased contractile force (Figure $4, C$ and D), as previously observed at the molecular and cellular levels with reduction in MyBP-C $(9,25,26)$. This faster contractile velocity was associated with a more rapid contractile deceleration time (Figure 4E). Thus, these findings suggest that heterozygous $M Y B P C 3$ mutations do not affect cellular contractility during early development when MyBP-C levels are compensated, but a severe reduction in MyBP-C in iPSCMs with homozygous mutations alters contractile kinetics.

Contractile dysfunction in homozygous MYBPC3-mutant iPSCMs is independent of calcium dynamics. Calcium mishandling has been thought to be a key feature of HCM (27), and we previously demonstrated calcium handling abnormalities specific to sarcomere mutation HCM using patient cardiac tissue samples, including samples with $M Y B P C 3$ mutations (28). Intracellular calcium measurements (with fura-2 indicator dye) in heterozygous and homozygous frameshift mutation lines demonstrated normal diastolic calcium level, normal peak calcium levels, and normal calcium transient kinetics (Supplemental Figure 7). These data indicate that calcium handling is not likely disrupted during early development in the presence of $M Y B P C 3$ mutations and that the contractile kinetic alterations observed in the homozygous frameshift mutant line are independent of calcium dynamics.

Global transcriptomic analysis demonstrates dysregulation of protein folding across all MYBPC3 loss-of-function mutation types. To agnostically identify dysregulated genes in MYBPC3-mutant lines, we performed RNASeq in the genome-edited lines compared with their original isogenic control and the CRISPR ${ }^{-}$isogenic control. The CRISPR-engineered isogenic lines were selected for this comparison to minimize irrelevant influences of genetic background when analyzing the entire transcriptome. This analysis included separately engineered iPSCM lines with allelic loss of function through different mechanisms, as well as a homozygous frameshift line to enable comparison with a condition of complete MyBP-C depletion. 

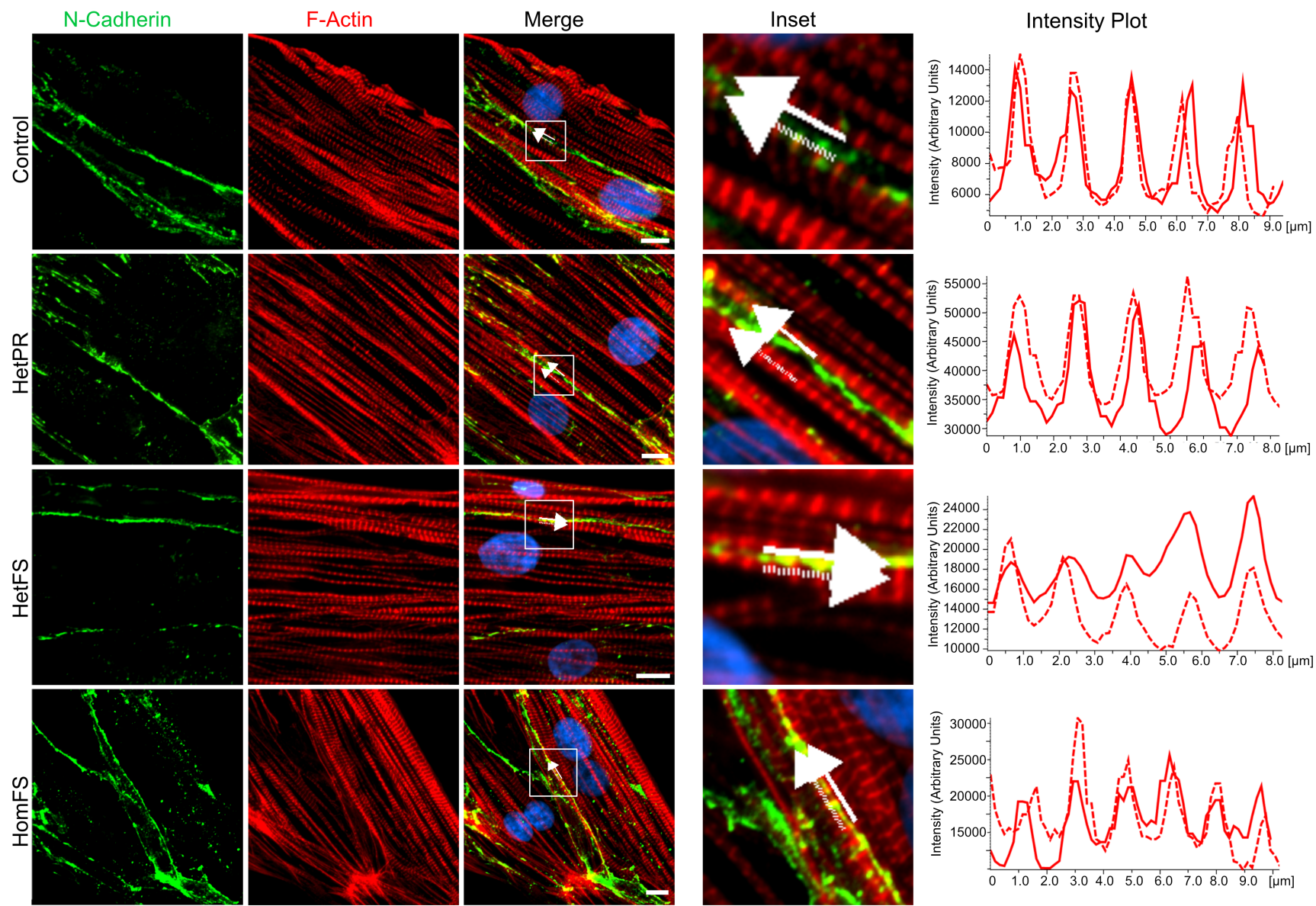

Figure 3. Intercellular alignment of iPSCMs in micropatterned 2D tissues is not disrupted by MYBPC3 mutations. Two-dimensional anisotropic cardiac tissues were generated by seeding iPSCMs onto row-micropatterned polydimethylsiloxane (PDMS) substrates. Because the row micropatterning induces cellular and myofibrillar alignment, the technique enables analysis of mutant impact on in vitro tissue formation. All the isogenic MYBPC3-mutant lines, including the homozygous line, formed well-aligned tissues, indicating that a tissue-level phenotype of disorganization does not occur in the isogenic MYBPC3 mutation iPSCM models. The intensity profiles depict alignment of adjacent iPSCMs in each 2D-tissue (cell-cell boundaries indicated by $\mathrm{N}$-cadherin) as evidenced by registration of Z-bands ( $\alpha$-actinin) in adjacent cells. The solid and dotted lines in the graphs indicate the intensity profiles from the solid and dotted line arrows in the images. Scale bar: $10 \mu \mathrm{m}$; inset: $\times 4$ magnification.

Global transcriptomic analysis identified only 59 dysregulated genes meeting statistical significance with multiple-testing adjustment among all MYBPC3 mutation groups $(N=4$ mutant lines, 20 total samples $)$ compared with controls ( $N=2$ mutant lines, 10 total samples). Gene Ontology (GO) analysis revealed an enrichment of 5 biologic processes, all of which are related to protein chaperones and quality control (Figure $5 \mathrm{~A}$ ). These results were driven by the 5 genes shown in Figure 5B. Of note, 2 of these genes, HSPA1A and HSPA1L, are paralogs adjacent to each other on chromosome 6, and the similar expression profiles among groups is suggestive of coregulation in this context. These genes express the Hsp70 and Hsp70-like proteins, respectively, and MyBP-C is a known client of Hsp70 family chaperones (29). Both HSPA1A and HSPA1L were downregulated similarly among the heterozygous lines. There was no difference in the transcriptional profile of protein quality control genes among the iPSC lines that either do or do not express transcripts containing PTCs, indicating that differential expression of these genes is likely not due to degradation of truncated peptides or NMD.

In addition, we performed prespecified pathway analysis for calcium-handling genes, NMD genes, and hypertrophy-associated genes. Further supporting an absence of calcium mishandling in the early developmental stage, we found no significantly dysregulated calcium-handling genes in either heterozygous or homozygous models (Figure 5C), including ATPA2A (Serca2) and CAMK2D, both of which are dysregulated in $\operatorname{HCM}$ patient cardiac tissue and in mouse models of $\operatorname{HCM}(28,30)$. We also found no significant differential expression of NMD genes in $M Y B P C 3$ mutation lines, including in frameshift mutation lines (in which NMD degrades mutant mRNA transcripts) versus promoter deletion or start site mutation lines 
A

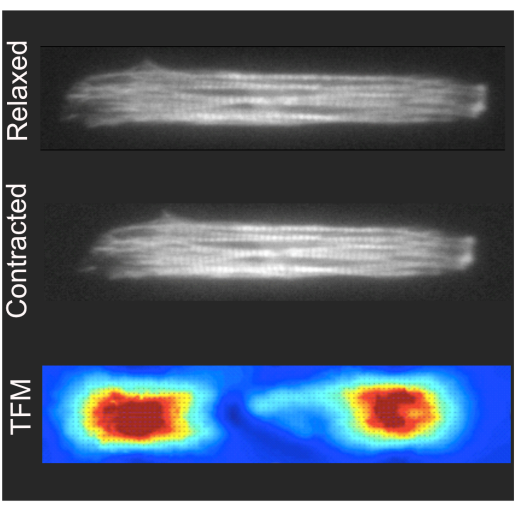

B

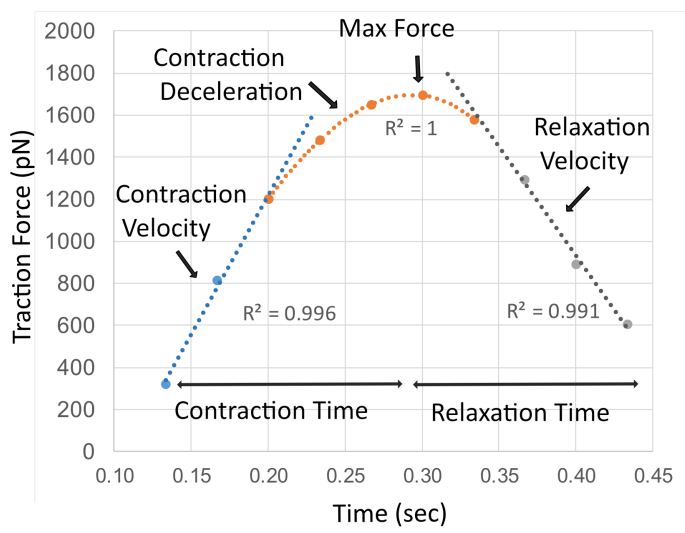

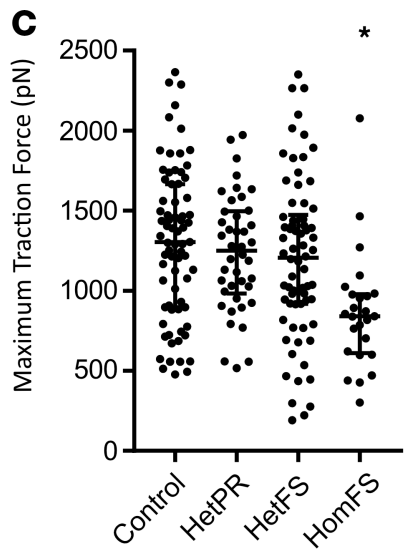

G

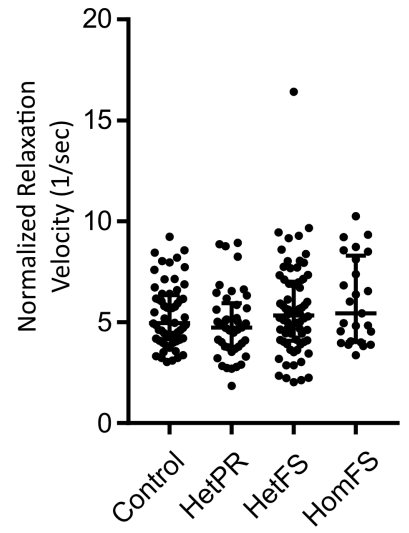

Figure 4. Contractile function is preserved in iPSCMs with heterozygous MYBPC3 mutations, whereas severe MyBP-C loss primarily dysregulates contractile deceleration. (A) Contractile quantification was performed in single 7:1 rectangular micropatterned iPSCMs on polyacrylamide gel substrates with physiologic elasticity $(8.7 \mathrm{kPa}$ ) that contained fluorescent microbeads. Tracking of fluorescent microbeads using traction force analysis software enabled calculation of traction force at each frame with 30-ms temporal resolution. F-actin live-cell staining enabled visualization of myofibrillar structure in each analyzed cell. (B) Representative time-force curve for an iPSCM depicts the analyzed contractile parameters. Contractile curves were partitioned into separate phases of contraction, as shown, to quantify each parameter depicted. (C) Maximum traction force was reduced in only HomFS. (D) Contraction time was reduced in HomFS. (E) Normalized maximum contraction velocity was increased in the HomFS only. (F) Contraction deceleration time was reduced only for the homozygous frameshift iPSCMs. (G) Normalized relaxation velocity was not different among any of the lines. All contractile parameters were analyzed using 1-way ANOVA with multiple-comparison significance determined by Dunnett's test $\left({ }^{*} P<0.05\right)$. The dot plots show bars at mean and standard deviations.

(in which mRNA loss of function occurs without NMD). This finding was consistent with the constant rate of NMD between iPSCMs with differing levels of mutant transcripts (i.e., heterozygous and homozygous), as described above (Figure 5D and Figure 1, C and D). Last, analysis of hypertrophy-associated genes showed no significant differential regulation among any MYBPC3-mutant line compared to controls (Figure 5E). Taken together, the RNA-Seq data support an immediate cellular consequence of MYBPC3 loss-offunction mutations on protein quality control gene dysregulation, while indicating that known HCM tissue calcium handling and hypertrophic gene dysregulation likely occur as a result of downstream remodeling.

$M y B P-C$ protein level is dynamically regulated to maintain steady-state equilibrium during myofibrillogenesis despite reduced synthesis rate. Although we previously reported reduced MyBP-C in patient cardiac tissue in HCM due to $M Y B P C 3$ mutations, in the PPSCM data presented here, we found that heterozygous $M Y B P C 3$ mutations did not result in a reduction in MyBP-C at day 25 after differentiation, despite persistently approximately $50 \%$ reduced mRNA levels. In a heterozygous truncating MYBPC3 mutation mouse model, protein-level MyBP-C was not reduced in unstressed mice, but haploinsufficiency developed during pressure overload-induced hypertrophic growth by transaortic constriction (15). Therefore, we hypothesized that reduced mRNA levels would be rate limiting for MyBP-C synthesis during active growth in the PSCM model and that MyBP-C levels might be restored through compensatory slowing of degradation. To test this hypothesis, we examined the appearance of isotopically labeled amino acids in proteins in iPSCMs by enriching the growth medium 
A Over-Represented in MYBPC3 Mutant Groups

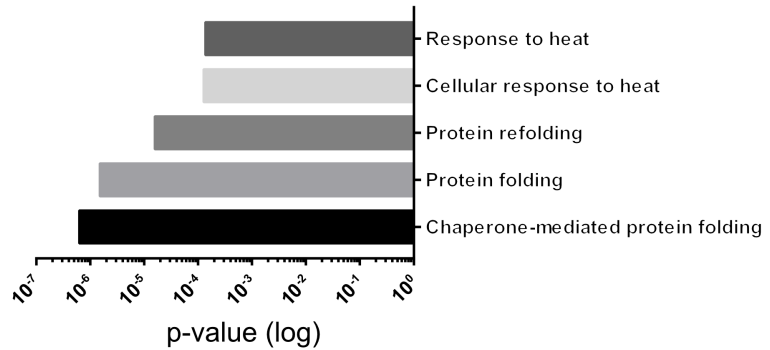

A Gene Ontology Categories

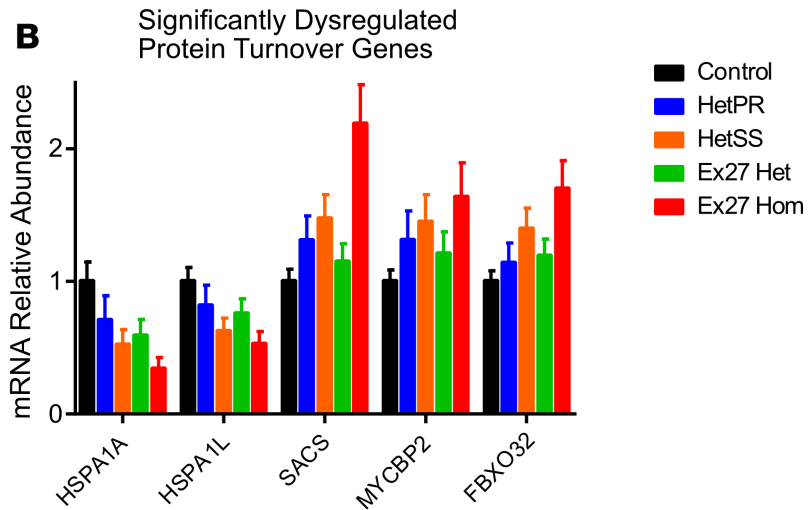

C

Calcium Handling

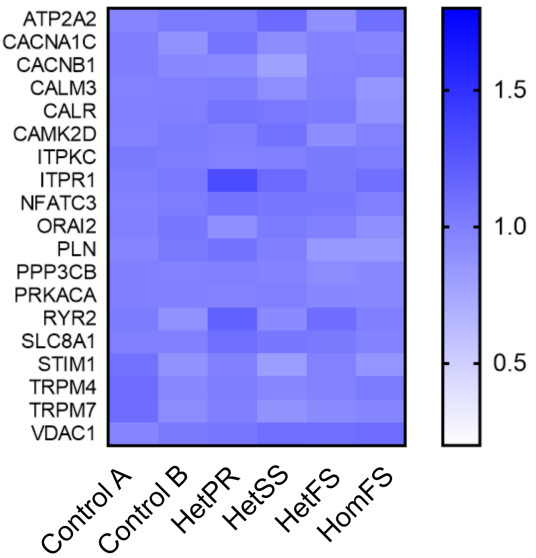

D

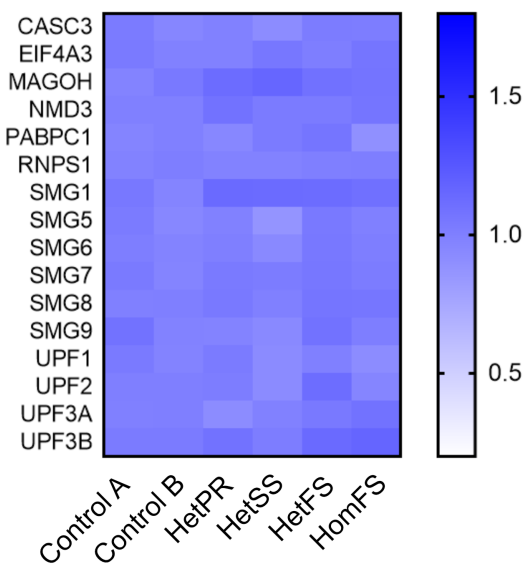

Figure 5. Global transcriptomic analysis demonstrates dysregulation of protein quality control across all MYBPC3 loss-of-function mutation types but no alterations in calcium-handling-, NMD-, or hypertrophy-associated gene sets. (A) Transcriptome-wide RNA-Seq analysis was performed and differentially expressed genes were tested for pathway enrichment with a Gene Ontology (GO) approach using GO-Seq, revealing 5 categories of genes significantly dysregulated in MYBPC3-mutant iPSCMs. Statistical significance was calculated using GO-Seq with multiple-testing correction for all biologic pathways tested. Isogenic MYPBC3-mutant iPSCM lines were used to avoid confounding with transcriptome-wide analysis, and 2 different control lines (original control iPSC line and nontargeted control iPSC line) were used ( $N=20$ total mutant samples; $N=10$ total control samples). (B) The top enriched GO categories identified were driven primarily by the same set of protein quality control genes. These genes, shown here, show a consistent pattern of dysregulation when compared individually against control samples and a stronger magnitude of effect in the homozygous frameshift line. The relative abundance was calculated as in Figure 1D, using DESeq2 estimates from negative binomial fits for each gene and with significance calculated by the Wald test. (C-E) Gene set enrichment analysis was also performed for prespecified, manually curated pathways, including NMD, calcium handling, and hypertrophy, none of which demonstrated evidence of dysregulation in the MYBPC3 gene-edited iPSCMs. Heatmaps of representative and highly expressed genes from these pathways are shown in C-E. Each gene's expression was calculated relative to the average of the controls and shown on a heatmap scale for fold change range of 0.2-1.8.

with $50 \% \mathrm{H}_{3}$-leucine 24 hours after replating cells from purified cultures at day 15 . Because myofibrils disassemble and reassemble following replating (31), we collected serial time point samples for mass spectroscopy analysis from days 16-21 (beginning 24 hours after replating) to take advantage of the period of active growth following replating. Because both gene-edited and patient iPSC lines had similar MyBP-C/myosin levels in day 25 samples (Figure 1B), the patient heterozygous frameshift mutation iPSCMs were used for this experiment to determine whether variability in the dynamic regulation of MyBP-C could be associated with the markedly different clinical disease severities between the 2 patients. New MyBP-C was synthesized at a slower rate in both heterozygous patient frameshift lines compared with controls (Figure 6A). However, degradation of MyBP-C was also slowed in the frameshift patient lines, resulting in a significantly smaller reduction in the starting pool of MyBP-C after 48 hours (Figure 6B). There was not a significant difference between the $2 \mathrm{HCM}$ lines in synthesis or degradation, despite the marked clinical variability between the 2 patients from whom these lines originated. The reduced degradation of MyBP-C enabled compensation in MyBP-C/ myosin stoichiometry (Figure 6C). Together with the mRNA quantification data, this finding indicates that reduced MYBPC3 mRNA levels are likely rate limiting for MyBP-C protein synthesis during active myocyte growth but that slowed MyBP-C degradation compensates for reduced synthesis. 
A
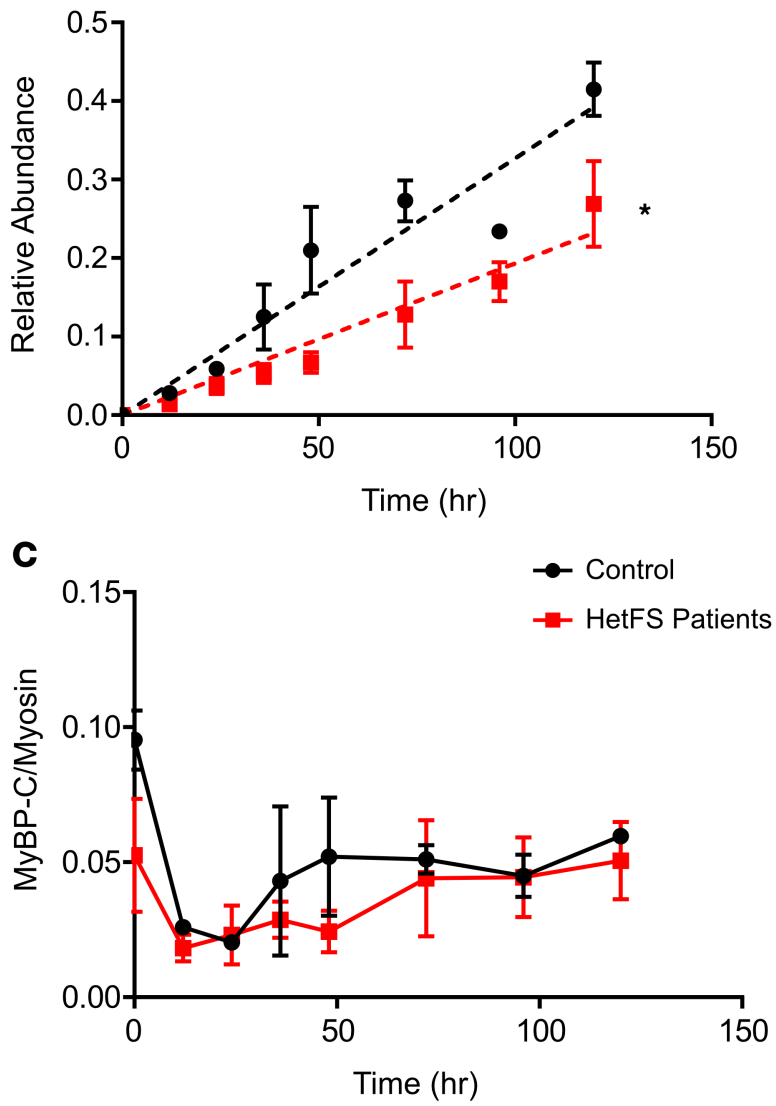

B

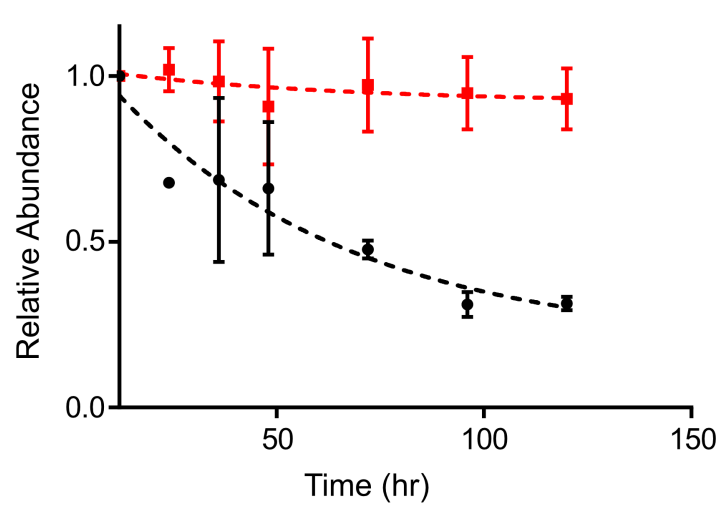

Figure 6. MyBP-C protein level is dynamically regulated to maintain steady-state equilibrium during myofibrillogenesis despite reduced synthesis rate. MyBP-C synthesis and degradation during cell growth following replating of purified iPSCMs was determined using mass spectroscopy analysis of isotopically labeled proteins, introduced through addition of deuterated leucine to the cell medium 24 hours after replating. Control iPSCMs were compared with iPSCM lines from patients with heterozygous frameshift MYBPC3 mutations (HetFS-P1 and HetFS-P2). (A) Newly synthesized MyBP-C was quantified by appearance of labeled MyBP-C peptides and appeared at a slower rate in frameshift MYBPC3-mutant iPSCMs compared with controls (analysis by comparison of the slopes of the linear regression fits, ${ }^{*} P<0.0001$ ). (B) MyBP-C degradation was quantified by measuring the relative reduction in unlabeled MyBP-C at each time point and was reduced in both HCM samples compared with the control samples at time points more than 48 hours $\left({ }^{*} P=0.001\right.$, Mann-Whitney). MyBP-C degradation was quantified beginning at 12 hours following labeling because a genotype-independent reduction in MyBP-C occurred in all samples in the first 12 hours following withdrawal of the $2 \%$ serum-containing replating medium. (C) The MyBP-C/myosin ratio was quantified at each time point as an indication of MyBP-C stoichiometry. In both control and HCM samples, MyBP-C/myosin reduced markedly in the first 12 hours following $2 \%$ serum withdrawal from the replating medium, prior to a return to normal levels, with no significant difference between control and HCM samples. Two samples were collected at each time point for controls and 2 samples each for the frameshift mutation lines. Data for each collection point is shown as the mean \pm range for all samples (range not shown when less than the size of the dot).

\section{Discussion}

Although MYBPC3 mutations are the most common genetic cause of familial HCM, the temporal sequence of events leading from the inciting mutation to pathologic hypertrophic remodeling has been elusive. In patient cardiac tissue, we previously showed an approximately $40 \%$ reduction in MyBP-C content, consistent with haploinsufficiency at the mature stage of HCM. Here, we take advantage of gene editing and the early developmental state of iPSC-derived cardiomyocytes to establish the regulatory levels at which $M Y B P C 3$ gene expression and MyBP-C protein homeostasis are maintained in the presence of PTC-causing MYBPC3 mutations. Importantly, we find that MYBPC3-mutant iPSCMs exhibit normal myofibrillogenesis and are prehypertrophic, even in the case of complete MyBP-C ablation. This result indicates that the iPSCM models can be used to identify the earliest cellular perturbations that occur as direct responses to $M Y B P C 3$ mutations. In this context, we find several primary immediate consequences of $M Y B P C 3$ mutations. First, we demonstrate that no innate compensation occurs at the $M Y B P C 3$ mRNA level, regardless of whether NMD is involved in allelic loss of function. Second, we find reduced MyBP-C synthesis in heterozygous mutant MYBPC 3 iPSCMs that is immediately compensated for by reduced MyBP-C degradation to dynamically attain normal levels relative 
to myosin at this early developmental stage. Third, using a transcriptome-wide approach, we find that protein folding-associated gene pathways are the only significantly dysregulated gene sets among MYBPC3-mutant cell lines. Finally, we find that MyBP-C protein-level compensation in heterozygous mutant iPSCMs prevented contractile dysfunction, whereas complete MyBP-C ablation resulted in contractile dysregulation.

Sarcomeric stoichiometry is known to be tightly maintained, but evidence for the specific mechanisms that regulate expression of individual sarcomere genes is limited $(14,32)$. We leveraged multiple gene-edited iPSC lines to interrogate whether cardiomyocytes detect and react to MYBPC3 mRNA loss, both when due to PTC-causing mutations and when due to allelic loss of function by other mechanisms (promoter deletion, translational start site disruption). We found that, regardless of the mechanism of $M Y B P C 3$ mRNA loss, there is no evidence of any compensatory transcriptional upregulation of $M Y B P C 3$. However, this evidence alone does not necessarily prove a haploinsufficiency mechanism because sarcomere gene mRNA levels may not necessarily be rate limiting for protein translation (14). Though cardiomyocytes are terminally differentiated cells, their constituent myofilament proteins, including MyBP-C, are in a state of constant protein turnover governed by synthesis and degradation rates. In the iPSCM model presented here, we found that MyBP-C abundance was not reduced relative to myosin in day 25 iPSCMs, despite reduced mRNA levels. Using a highly quantitative mass spectroscopy approach, we found that this result was reproducible in not only the gene-edited iPSC lines but also in previously published iPSC lines from HCM patients with both mild and very severe pathologic hypertrophy (13). Taken together with results from an agnostic RNA-Seq analysis approach that identified protein quality control gene sets as the only differentially regulated pathway, we hypothesized that (a) reduced MYBPC3 mRNA is rate limiting for normal MyBP-C synthesis and (b) a compensatory slowing of MyBP-C degradation is responsible for the equivalency in net MyBP-C protein. To support this hypothesis, we demonstrated that both MyBP-C synthesis and degradation rates were reduced in the HCM patient lines using isotope labeling. Although we had further hypothesized that there may have been a difference in synthesis and degradation rates between the 2 HCM patient iPSC lines that would correlate with disease severity, we showed no difference between the 2 patient lines. This latter finding indicates that the (presumed) genetic modifiers in the more severely affected patient likely relate to differences in downstream remodeling pathways.

Knowledge of the regulatory mechanisms for MyBP-C turnover has been limited. In a prior study, we used an agnostic mass spectroscopy analysis of coimmunoprecipitated proteins to identify the binding partners of MyBP-C, which revealed that the Hsp70 protein family was critical for MyBP-C clearance. Here, we used an RNA-Seq approach, utilizing 4 gene-edited MYBPC3 mutation lines and 2 control lines (including a nontargeted control clone), all with the same genetic background. We identified a limited list of dysregulated genes common to all mutation groups. We found that protein quality control genes were the primary group of dysregulated genes, including HSPA1A (encoding Hsp70). Moreover, the fact that $H S P A 1 L$, a paralog of HSPA1A adjacently situated on chromosome 6, was also significantly dysregulated in the same direction among all $M Y B C P 3$-mutant samples strongly suggests cis regulation of both HSPA1A and HSPA1L through a transcriptional mechanism responsive to MyBP-C protein-level aberration. Both the downregulation of MyBP-C degradation rate and the altered protein turnover gene regulation indicate that MyBP-C homeostasis is achieved through slowing of protein clearance. The significance of upregulation of other protein turnover gene transcripts (Figure 5) was not immediately clear but could implicate either compensatory effects to maintain MyBP-C levels at equilibrium or secondary effects of Hsp70 downregulation. These genes include $S A C S$ (encodes sacsin, which interacts with Hsp70 and the ubiquitin-proteosome system), MYCBP2 (encodes the E3 ubiquitin-protein ligase MYCBP2), and FBXO32 (encodes the E3 ubiquitin ligase atrogin-1). Altered protein quality control has been demonstrated previously in myocardial samples from both patients with HCM and mouse models (33-35). In the case of MYBPC3 loss-of-function mutations, identifying specific protein quality control proteins responsible for this compensatory response to maintain protein homeostasis versus those that are nonspecifically dysregulated as a secondary effect of chronic hypertrophic remodeling will be an important focus of future investigation.

The temporal relationship of HCM-causing mutations and the development of HCM has been challenging to unravel. Biochemical and molecular studies of human HCM hearts have been performed in surgical heart tissue samples, for which the hypertrophic disease is already manifest, and, therefore, causality of detected abnormalities is difficult to elucidate. A range of abnormalities have been described in these late-stage disease tissues, including myofibrillar disarray, contractile abnormalities, and calcium mishandling (8, 28, 36-38). 
We find here that isogenic iPSCMs with $M Y B P C 3$ loss-of-function mutations exhibit normal myofibrillogenesis, even in a state of severe MyBP-C depletion. This finding is in contrast to the case of myosin depletion, which completely prevents myofibrillogenesis (31), but is consistent with studies from mice with a homozygous MYBPC3 splice mutation causing near-complete loss of MyBP-C (39). Moreover, neither heterozygous nor homozygous MYBPC3-mutant iPSCMs exhibited myofibrillar hypertrophy at this developmental stage, either when measured directly with a precise cellular-based assay or when measured indirectly through transcriptome-based pathway analysis. In this context, we find that MYBPC3-mutant iPSCMs do not demonstrate the myofibrillar disarray or calcium handling evident in human heart tissue, indicating that these phenomena likely occur indirectly, through downstream remodeling. Taken together, our results are consistent with a haploinsufficiency mechanism in which an early quiescent stage of compensated MyBP-C levels (despite reduced synthesis) precedes conversion to an uncompensated stage of overt protein-level MyBP-C reduction at a later point (9), which then causes contractile dysregulation, finally leading to the remodeling pattern characterized by hypertrophy, myofibrillar disarray, and calcium mishandling. These results also may reconcile the findings by Barefield et al. (15), who found that heterozygous frameshift MYBPC3-mutant adult mice only developed a relative MyBP-C protein reduction following transaortic constriction, a procedure that dramatically increases hemodynamic stress to induce a rapid hypertrophic response. The studies presented here cannot distinguish whether (a) the observed cellular MyBP-C stoichiometry is achieved at the expense of dysregulation in protein turnover that itself may contribute to hypertrophic remodeling or (b) cellular MyBP-C stoichiometry is preserved innately in the embryonic/fetal stage but this capacity is later diminished during postnatal growth, leading to haploinsufficiency-driven contractile dysfunction that then induces hypertrophic growth.

Contractile dysregulation is clearly a consequence of MyBP-C reduction, both with partial reduction and complete loss $(9,40-42)$. We showed here that compensatory mechanisms that maintain MyBP-C levels in heterozygous mutant iPSCM models are protective against contractile dysfunction at this developmental stage, and this result was the same in 2 heterozygous gene-edited MYBPC3-mutant iPSC lines (frameshift and promoter deletion). To ensure rigor and reproducibility of these experiments, we used live-cell actin staining to verify that the single micropatterned iPSCMs had similar myofibrillar development - importantly, this method limited confounding variability among cells within and across batches (Supplemental Figure 7, B and C). In contrast, we found clear evidence of contractile dysregulation in the homozygous $M Y B P C 3$-mutant line. Contractile time was reduced, with a strikingly faster time for contractile deceleration. This finding is consistent with molecular studies from adult HCM hearts lacking the full complement of MyBP-C that demonstrate enhanced contractile sliding velocities within the C-zone, when compared with donor controls $(2,9)$. Faster contractile kinetics, particularly in the late phase of contraction, have also been reported in engineered heart tissues from mice with MyBP-C ablation (26).

Prior iPSCM studies of $M Y B P C 3$ have reported both similar and distinct findings from aspects of our work. Birket et al. reported a protein-level reduction in MyBP-C by Western blot and a reduced contractile force in single iPSCMs from these cells (13). To enable direct comparison to these findings, the Mummery lab (Leiden University, Leiden, the Netherlands) contributed these same patient lines (the HetFS-P1 and HetFS-P2 iPSC lines). When we applied our mass spectroscopy quantification approach to these patient lines, we found no difference in MyBP-C relative to myosin at steady state and correspondingly did not find a lower maximum contractile force. These findings could be due to the media conditions, timing of analysis, or developmental stage of the iPSCMs. We used a single-cell traction force microscopy approach with additional controls for geometric shape (7:1 rectangular micropatterns as opposed to row micropatterns) and myofibrillar density (using concurrent live-cell actin imaging) that might also account for differences. Our finding of restoration of MyBP-C protein-level stoichiometry is similar to the recently reported findings by Seeger et al. (11), who also reported no difference in maximal contractile force in engineered cardiac tissues derived from a heterozygous MYBPC3-mutant iPSCM line containing a nonsense mutation, consistent with our results. However, in contrast to that work, we did not find evidence of dysregulation of calcium handling or NMD in the several MYBPC3-mutant iPSC lines that we generated, and we found a constant rate of NMD between heterozygous and homozygous C-terminal mutation lines. Although it remains possible that mutation locus may variably affect pathogenic mechanisms, possibly explaining these differences, we did not find evidence of distinct disease mechanisms among the promoter deletion, translational start site deletion, or frameshift mutation lines. Our finding of a reduced maximum contractile force in the homozygous mutant $M Y B P C 3$ model is consistent with the report from Ma et al. (43), who showed reduced force generation in homozygous truncating $M Y B P C 3$-mutant iPSCMs using cardiac tissue 
constructs on filamentous 3D matrices - interestingly, that study found a reduction in contractile force only in the setting of mechanical afterload (high fiber stiffness), similar to the biomechanical environment of our approach, in which single iPSCMs contracted against the inherent physiologic level of stiffness of engineered polyacrylamide gels. We additionally found a faster contractile time, particularly in the deceleration phase close to peak contraction, similar to findings from engineered heart tissue from mice with MyBP-C ablation (26). Taken together, the finding of workload-dependent contractile dysfunction in the homozygous mutant $M Y B P C 3$ lines, distinct from the hypercontractility reported in unloaded homozygous mouse mutant $M Y B P C 3$ cardiomyocytes (40), may have important implications for both disease modeling and targeting of $M Y B P C 3$-related contractile dysfunction with novel therapeutic approaches.

In conclusion, we have harnessed patient and gene-edited MYBPC3-mutant iPSCMs to deeply investigate the effects of $M Y B P C 3$ mutations in the early developmental stage modeled by iPSCMs. We show compelling evidence that the common mechanism shared by PTC-causing MYBPC3 mutations is loss of function due to reduced MyBP-C synthesis as a consequence of an uncompensated reduction in $M Y B P C 3$ mRNA. We also show that a key distinction of iPSCMs compared with HCM patient heart tissue is the innate capacity to attain normal MyBP-C levels through a compensatory slowing of MyBP-C degradation - a capacity that is presumably lost by the time HCM has developed. This work therefore suggests an optimistic concept that modulation of the MyBP-C degradation pathway in patients with HCM to maintain normal MyBP-C levels beyond early development could be a novel therapeutic avenue to prevent the development of contractile dysfunction and adverse cardiac remodeling.

\section{Methods}

iPSC and cardiomyocyte cell culture. Control iPSCs (DF19-9-11) were obtained from the WiCell repository, and the originating clone from which gene-edited lines were generated is referred to as control A. A second subclone derived from the original DF19-9-11 line from a genome-editing experiment, but confirmed to have no mutation at the targeting site, served as control B. The DF19-9-11 line was selected because it is a well-validated control cell line with similar calcium-handling properties as other control cell lines (44, 45). HCM patient lines were obtained from Leiden University (Leiden, the Netherlands) $(13,16)$. All iPSCs were cultured in mTeSR1 and differentiated using WNT modulation (46). iPSCs were verified to be free of mycoplasma contamination using MycoAlert (Lonza). iPSC-derived cardiomyocytes were purified with signal regulatory protein- $\alpha$ (SIRPA) selection using magnetic beads at day 15 of differentiation (47). SIRPA-sorted cardiomyocytes were more than $90 \%$ pure by immunofluorescence ( $\alpha$-actinin) and by FACS (TNNT2). Following SIRPA purification, iPSCMs were plated at 600,000 cells per well in 12-well plates on PDMS substrates (47). iPSCMs were maintained in RPMI with B27 supplement until collection at day 25 for RNA and protein samples. For the myofibril assembly and aligned 2D tissue assays, purified iPSCMs were replated onto micropatterned PDMS substrates (see below) and cultured for an additional 8 days prior to fixation. For the contractile assay, purified iPSCMs were initially plated as monolayers following SIRPA purification, cultured for 6 days, and then replated onto micropatterned hydrogels at day 25. For the MyBP-C synthesis experiment, iPSCMs were purified using metabolic selection and frozen in Cryostor (MilliporeSigma) at $-80^{\circ} \mathrm{C}$ at day $15(48,49)$.

Gene editing. All potential gRNAs with target MYBPC3 regions of interest were first identified. Potential gRNA sequences with highest stringency for target sites (the first 13 nucleotides) were then selected by entering all potential sequences into the National Center for Biotechnology Information's (NCBI) nucleotide BLAST (http://www.ncbi.nlm.nih.gov/blast/) and identifying gRNAs with minimal or no other sequence matches in the reference human genome (Supplemental Table 1). Guide RNA constructs were synthesized as gBlocks (Integrated DNA Technologies) as described and cloned using Zero Blunt TOPO cloning (Thermo Fisher Scientific) (50). Cas9-GFP was obtained from the Musunuru laboratory's repository via Addgene (plasmid 44719) (51). Cas9-GFP and gRNAs were transfected into iPSCs, selected by FACS to obtain single cell-derived clones, and genotyped (primer sequences in Supplemental Table 2) (52). Differentiations of gene-edited iPSCs were performed within 15 passages of initial gene editing.

Microcontact printing. For single-cell micropatterning, a chrome mask with grids of 7:1 aspect ratio, 1750$\mu \mathrm{m}^{2}$ area rectangles was fabricated, then used to generate a silicon wafer with micropatterned spin-coated SU-8 using the Lurie Nanofabrication Facility (University of Michigan). PDMS stamps were then molded with 10:1 (base/curing agent) Sylgard 184 (Corning). For row micropatterning, PDMS stamps with 30- $\mathrm{mm}$ wide rows with $3-\mu \mathrm{m}$ gaps were fabricated. For the myofibril assembly assay, structural quantification, and 
2D tissue quantification, microcontact printing was performed on PDMS sheeting (Specialty Manufacturing, Inc.) using stamps that were first incubated with 1:25 diluted 0.1\% human serum-derived fibronectin (MilliporeSigma). Substrates were incubated for 30 minutes with 1\% pluronic F127 (MilliporeSigma) and then rinsed 4 times with PBS prior to use. For contractility studies, microcontact printing was performed on polyacrylamide gels, as described below.

Myofibrillar assembly and aligned 2D tissue assays. Purified iPSCMs were plated on single-cell or row-patterned PDMS substrates, and medium was changed every 2 days with medium pre-equilibrated in the cell culture incubator before fixation at 8 days with 4\% paraformaldehyde. Preparations were treated with $0.1 \mathrm{M} \mathrm{NH}_{4} \mathrm{Cl}$ for 10 minutes, then $10 \mathrm{mM}$ sodium citrate with $0.05 \%$ Tween-20 for 15 minutes. For the myofibrillar assay, iPSCMs were incubated with antibodies against MyBP-C (gift from Samantha Harris, University of Arizona, Tucson, Arizona, USA) and $\alpha$-actinin (MilliporeSigma), at 1:1000 dilution in 5\% goat serum in PBS with $0.2 \%$ Triton X-100 overnight at $4^{\circ} \mathrm{C}$. For $2 \mathrm{D}$ tissue quantification, iPSCMs were incubated with antibodies against N-cadherin (1:200, BD Biosciences) and $\alpha$-actinin (1:1000, MilliporeSig$\mathrm{ma}$ ). Microscope slides were labeled with a blinded numbering system and saved for batched analysis.

For myofibrillar analysis, images were saved for at least 100 cells per genotype. Only cells that filled more than $70 \%$ of the micropatterned areas with myofibrils reaching end to end of the long axis were imaged from at least 5 batches. Myofibrillar counts were performed blinded to genotype by counting the number of myofibrillar bundles that traversed the long axis. For $2 \mathrm{D}$ aligned tissue analysis, images were obtained from at least 5 tissues from at least 3 differentiation batches. Blinded images were examined for evidence of disordered myofibrillar alignment and evidence of Z-disk misregistration at cell-cell borders.

Contractility assay. Polyacrylamide hydrogels were fabricated onto glass coverslips, as described previously $(23,53)$. Polyacrylamide gels were polymerized using an $18-\mathrm{mm}$ top coverslip to sandwich a $25-\mu \mathrm{L}$ hydrogel volume in the center of the bottom coverslip before assembly in a culture dish. The hydrogel method used functionalization of the polyacrylamide gel to bind matrix proteins as described (53). Adherence of matrix proteins to hydrogels was confirmed using fluorescently labeled gelatin (Supplemental Figure 7A). Hydrogels contained 0.2- $\mu \mathrm{m}$ diameter FluoSpheres (Thermo Fisher Scientific). Purified day 25 iPSCMs (as above) were replated at $6000 / \mathrm{cm}^{2}$ density. iPSCMs were cultured for 6-7 days on these devices before microscopy at days 31-32. Because RPMI contains a subphysiologic level of calcium (0.4 mM), RPMI was blended 50:50 with DMEM to achieve $1.10 \mathrm{mM}$ calcium on day 1 following replating and then 25:75 with DMEM to achieve $1.45 \mathrm{mM}$ calcium on day 3 following replating (1X B27 supplement, Gibco, Thermo Fisher Scientific). In our experience, single iPSCMs at the $0.4-\mathrm{mM}$ calcium level with typical RPMI did not demonstrate enough contractile force to be reliably quantified. All iPSCMs were labeled for F-actin using the live-cell dye SiR-Actin (Cytoskeleton, Inc.) the day prior to imaging $(0.5 \mu \mathrm{M}$ of SiR-Actin for 1 hour) to enable simultaneous visualization of sarcomeres. All media were pre-equilibrated to the incubator environment prior to medium changes. Microscopy was performed in a microscope stage top incubator at $37^{\circ} \mathrm{C}$ and $5 \%$ $\mathrm{CO}_{2}$ to acquire videos of single, spontaneously contracting iPSCMs and the underlying layer of fluorescent microbeads at 30 frames per second (fps). Videos were acquired of myofibrils at $200 \mathrm{fps}$. To account for the variability in myofibrillar density, blinded videos of F-actin-labeled cells were analyzed using a binary mask in Nikon Elements, and only iPSCMs with a myofibrillar density more than $40 \%$ were selected for subsequent analysis. Traction force analysis was performed using the method of Han et al. (24). Time-force data were exported to Microsoft Excel for curve fitting (as in Figure 4B) and extraction of contractile kinetic data.

Intracellular calcium quantification. iPSCMs were replated onto $8.7-\mathrm{kPa}$ polyacrylamide gels fabricated as for the contractility assay (above), but with nonpatterned fibronectin, to enable measurements in multicellular 2D iPSCM tissues. Calcium measurements were performed on the $8.7-\mathrm{kPa}$ gels to enable physiologic fractional shortening during contractions, rather than isometric contractions on glass. iPSCMs were labeled for F-actin as above, loaded with $1 \mu \mathrm{M}$ fura-2 (Thermo Fisher Scientific) for 10 minutes, rinsed with $250 \mu \mathrm{M}$ probenecid (MilliporeSigma) in HBSS for 5 minutes, changed to pre-equilibrated 25:75 RPMI/DMEM medium, and then incubated for 30 minutes prior to imaging. Microscopy was performed at $37^{\circ} \mathrm{C}$ and $5 \% \mathrm{CO}_{2}$. iPSCMs were paced at $0.75 \mathrm{~Hz}$ (IonOptix Myopacer). iPSCM 2D monolayer tissues consisting of 4-10 cardiomyocytes were imaged through a Nikon Super Fluor 40× objective. The emitted light intensity was reported as fura- 2 ratios. F-actin was also imaged (far red) to enable identification of iPSCMs with robust myofibers for calcium measurements (Supplemental Figure 8).

Transcriptome quantification. Per genotype, 5 samples were assayed from at least 3 differentiation batches per line. Only samples with a spontaneous contraction frequency between 0.3 and $0.6 \mathrm{~Hz}$ were selected. RNA 
samples were processed in a single batch using the RNeasy kit (Qiagen). Only samples with an RNA abundance more than $25 \mathrm{ng} / \mu \mathrm{L}, 260 / 280$ ratio more than 1.9 (by NanoDrop, Thermo Fisher Scientific), and RNA integrity number greater than 8.0 were used for RNA library preparation. A stranded and barcoded RNA library was prepared and sequenced using an Illumina HiSeq 4000 with samples multiplexed across lanes to obtain a final read depth of about $35 \times 10^{6}$ reads per sample by the University of Michigan DNA Sequencing Core. Reads were aligned to the genome using STAR (54). Lowly expressed genes were filtered, leaving a data set of 16,819 genes. Reads per gene were normalized to the library size for each sample using DESeq2 (55). RUV-Seq was then used to control for additional technical factors using a robust housekeeping control set with minimal expression differences across multiple tissue types $(56,57)$. Relative abundance for each gene was determined using dispersion estimates for each gene fit to a binomial generalized linear model with DESeq2 (55). The Wald test with multiple-testing correction on 16,819 genes tested was performed to determine statistical significance (adjusted $P$ value < 0.10) (55). GO-Seq was used to test for enrichment of biologic pathways among the top 100 dysregulated genes with multiple-testing correction for all pathways tested. Prespecified gene sets for pathways with hypothesis-driven questions (i.e., sarcomere, NMD, calcium-handling, and hypertrophy gene sets) were manually curated and tested by gene set enrichment analysis. These data were deposited in the NCBI's Gene Expression Omnibus database (GEO accession GSE140965) (58).

Mass spectroscopy. iPSCM cell pellets ( 200,000 cells) were rinsed with PBS containing a protease inhibitor cocktail (Roche) and frozen at $-80^{\circ} \mathrm{C}$. Pelleted iPSCMs were thawed and solubilized in $75 \mu \mathrm{L} 0.1 \%$ RapiGest SF Surfactant (Waters Corporation) at $50^{\circ} \mathrm{C}$ for 1 hour. Subsequent processing of samples is described in the expanded Supplemental Methods. Peptides were separated by liquid chromatography, and their abundances were measured by mass spectrometry as detailed in the Supplemental Methods. MyBP-C relative abundance was quantified by using the average of the counts for the 3 most abundant MyBP-C peptides ([R].LTVELADHDAEVK.[W], [K].VIDVPDAPAAPK.[I], [R].IPDTIVVVAGNK.[L]) normalized to the average of the counts for the 3 most abundant myosin peptides shared between the $\alpha$ - and $\beta$-isoforms ([K].DTQIQLDDAVR.[A], [K].EALISQLTR.[G], [R].AQLEFNQIK.[A]). Using the most abundant peptides for both MyBP-C and myosin resulted in the expected molar ratio of about 0.10 in control samples, similar to our previous report from human heart tissue (9).

Protein synthesis and degradation were quantified by determining the incorporation of stable isotopelabeled amino acids into proteins using mass isotopomer analysis (59). Cryopreserved iPSCMs from 3 different batches for each of the lines tested (control, HetFS-P1, HetFS-P2) were thawed and pooled together for each line at the beginning of the experiment to synchronize all subsequent time points. Then, 24 hours after plating, the cells were rinsed with PBS, and the medium was changed to identical medium except without the $2 \%$ FBS present in the replating medium and with the addition of deuterated leucine (L-leucine-5,5,5-d3, MilliporeSig$\mathrm{ma})$ at an equivalent concentration of standard RPMI $(0.05 \mathrm{mg} / \mathrm{mL})$ to attain a final 50:50 ratio of labeled to native leucine isotopes. Duplicate samples were collected at each of the designated time points for each cell line, rinsed with $\mathrm{PBS}$ containing protease inhibitors (Roche), and frozen at $-80^{\circ} \mathrm{C}$ until processing. The samples were prepared and analyzed by liquid chromatography-mass spectometry (LCMS). The abundance of each mass isotopomer in the LCMS peaks of interest was determined using Pinpoint Software (Thermo Fisher Scientific), and distribution analysis was performed using Excel (Microsoft). The MyBP-C M+3 and M+0 (unlabeled) isotopomers were then quantified for each time point as described in the Supplemental Methods.

Coimmunoprecipitation and Western blots were performed to detect the presence of FLAG-MyBP-C in the heterozygous mutant FLAG-tagged iPSCMs (Supplemental Figure 5) essentially as described (29). Western blotting to detect the presence/absence of MyBP-C in the homozygous MYBPC3 promoter deletion line was performed as described, using the same MyBP-C antibody as for immunofluorescence imaging as above (6).

Statistics. Values are expressed as mean \pm SEM unless otherwise indicated. Normality was determined by the Shapiro-Wilk test. Normally distributed data were analyzed by 1-way ANOVA, and Dunnett's test was used for multiple comparisons if the overall result was statistically significant. Pairwise significance was tested if the overall value was $P<0.05$. Statistical significance for all tests was defined by a 2 -sided value of $P<0.05$. Statistical analysis of RNA-Seq data, including multiple-testing correction, was performed using DESeq2 (55). Statistical analysis was performed with GraphPad Prism except for transcriptomic analysis, for which Prism was used only for creating figures.

Study approval. The study was approved by the University of Michigan Human Pluripotent Stem Cell Research Oversight Committee. Human iPSCs obtained from patients at Leiden University were obtained with patient consent as previously published (16). 


\section{Author contributions}

ASH and SMD conceived the study and designed the experiments. ASH analyzed data, directed the study, and wrote the manuscript. SMD revised the manuscript. VTT, SF, MW, AA, and AHW conducted experiments. TSO conducted mass spectroscopy experiments and analyzed data. LML assisted in the design and fabrication of micropatterning masks. EDS analyzed data. XWW provided guidance in RNA-Seq analysis. JAS provided guidance in genome engineering and manuscript revision. APL assisted in the design of micropatterning masks and experiments and manuscript revision. MJP provided guidance in designing the mass spectroscopy experiments, directed the mass spectroscopy data acquisition and analyses, and revised the manuscript.

\section{Acknowledgments}

This work was funded by the NIH (K08HL130455 to ASH), by the American Heart Association Grant-inAid (to SMD), the Alfred A. Taubman Medical Research Institute (to SMD), the Liz and Eric Lefkofsky Scholar Award (to SMD), and the Children's Cardiomyopathy Foundation (to SMD). We thank Christine Mummery for contributing patient iPSC lines. We thank Kevin Kit Parker and Thomas Grevesse for initial training in the polyacrylamide gel micropatterning techniques. We thank Sangyoon Han for adapting the traction force analysis software he developed and for his assistance in implementing the analysis.

Address correspondence to: Adam S. Helms, 7220 MSRB3, 1150 W. Medical Center Drive, Ann Arbor, Michigan 48109, USA. Phone: 734.764.4500; Email: adamhelm@umich.edu.

SMD's present address is: Department of Medicine, Division of Cardiovascular Medicine, and Department of Genetics, University of Pennsylvania, Philadelphia, Pennsylvania, USA.

1. Ho CY, et al. Genotype and lifetime burden of disease in hypertrophic cardiomyopathy: insights from the Sarcomeric Human Cardiomyopathy Registry (SHaRe). Circulation. 2018;138(14):1387-1398.

2. Previs MJ, Beck Previs S, Gulick J, Robbins J, Warshaw DM. Molecular mechanics of cardiac myosin-binding protein C in native thick filaments. Science. 2012;337(6099):1215-1218.

3. Previs MJ, et al. Myosin-binding protein C corrects an intrinsic inhomogeneity in cardiac excitation-contraction coupling. Sci Adv. 2015;1(1):e1400205.

4. Zahka K, et al. Homozygous mutation of MYBPC3 associated with severe infantile hypertrophic cardiomyopathy at high frequency among the Amish. Heart. 2008;94(10):1326-1330.

5. Xin B, Puffenberger E, Tumbush J, Bockoven JR, Wang H. Homozygosity for a novel splice site mutation in the cardiac myosin-binding protein C gene causes severe neonatal hypertrophic cardiomyopathy. Am J Med Genet A. 2007;143A(22):2662-2667.

6. Helms AS, et al. Sarcomere mutation-specific expression patterns in human hypertrophic cardiomyopathy. Circ Cardiovasc Genet. 2014;7(4):434-443.

7. Marston S, et al. Evidence from human myectomy samples that MYBPC3 mutations cause hypertrophic cardiomyopathy through haploinsufficiency. Circ Res. 2009;105(3):219-222.

8. van Dijk SJ, et al. Cardiac myosin-binding protein C mutations and hypertrophic cardiomyopathy: haploinsufficiency, deranged phosphorylation, and cardiomyocyte dysfunction. Circulation. 2009;119(11):1473-1483.

9. O'Leary TS, Snyder J, Sadayappan S, Day SM, Previs MJ. MYBPC3 truncation mutations enhance actomyosin contractile mechanics in human hypertrophic cardiomyopathy. J Mol Cell Cardiol. 2019;127:165-173.

10. Monteiro da Rocha A, et al. Deficient cMyBP-C protein expression during cardiomyocyte differentiation underlies human hypertrophic cardiomyopathy cellular phenotypes in disease specific human ES cell derived cardiomyocytes. J Mol Cell Cardiol. 2016;99:197-206.

11. Seeger T, et al. A premature termination codon mutation in MYBPC 3 causes hypertrophic cardiomyopathy via chronic activation of nonsense-mediated decay. Circulation. 2019;139(6):799-811.

12. Prondzynski M, et al. Evaluation of MYBPC3 trans-splicing and gene replacement as therapeutic options in human iPSC-derived cardiomyocytes. Mol Ther Nucleic Acids. 2017;7:475-486.

13. Birket MJ, et al. Contractile defect caused by mutation in MYBPC3 revealed under conditions optimized for human psc-cardiomyocyte function. Cell Rep. 2015;13(4):733-745.

14. Lewis YE, Moskovitz A, Mutlak M, Heineke J, Caspi LH, Kehat I. Localization of transcripts, translation, and degradation for spatiotemporal sarcomere maintenance. J Mol Cell Cardiol. 2018;116:16-28.

15. Barefield D, et al. Haploinsufficiency of MYBPC3 exacerbates the development of hypertrophic cardiomyopathy in heterozygous mice. J Mol Cell Cardiol. 2015;79:234-243.

16. Dambrot C, Braam SR, Tertoolen LG, Birket M, Atsma DE, Mummery CL. Serum supplemented culture medium masks hypertrophic phenotypes in human pluripotent stem cell derived cardiomyocytes. J Cell Mol Med. 2014;18(8):1509-1518.

17. Davies MJ. The current status of myocardial disarray in hypertrophic cardiomyopathy. Br Heart J. 1984;51(4):361-363.

18. Bray MA, Sheehy SP, Parker KK. Sarcomere alignment is regulated by myocyte shape. Cell Motil Cytoskeleton. 2008;65(8):641-651.

19. Ribeiro AJ, et al. Contractility of single cardiomyocytes differentiated from pluripotent stem cells depends on physiological shape and substrate stiffness. Proc Natl Acad Sci USA. 2015;112(41):12705-12710.

20. Sheehy SP, Pasqualini F, Grosberg A, Park SJ, Aratyn-Schaus Y, Parker KK. Quality metrics for stem cell-derived cardiac myo- 
cytes. Stem Cell Reports. 2014;2(3):282-294

21. Pasqualini FS, Sheehy SP, Agarwal A, Aratyn-Schaus Y, Parker KK. Structural phenotyping of stem cell-derived cardiomyocytes. Stem Cell Reports. 2015;4(3):340-347.

22. Kalstrup T, Blunck R. Reinitiation at non-canonical start codons leads to leak expression when incorporating unnatural amino acids. Sci Rep. 2015;5:11866.

23. Grevesse T, Versaevel M, Gabriele S. Preparation of hydroxy-PAAm hydrogels for decoupling the effects of mechanotransduction cues. J Vis Exp. 2014;(90):51010.

24. Han SJ, Oak Y, Groisman A, Danuser G. Traction microscopy to identify force modulation in subresolution adhesions. Nat Methods. 2015;12(7):653-656.

25. Stelzer JE, Fitzsimons DP, Moss RL. Ablation of myosin-binding protein-C accelerates force development in mouse myocardium. Biophys J. 2006;90(11):4119-4127.

26. de Lange WJ, Grimes AC, Hegge LF, Ralphe JC. Ablation of cardiac myosin-binding protein-C accelerates contractile kinetics in engineered cardiac tissue. J Gen Physiol. 2013;141(1):73-84.

27. Ashrafian H, McKenna WJ, Watkins H. Disease pathways and novel therapeutic targets in hypertrophic cardiomyopathy. Circ Res. 2011;109(1):86-96.

28. Helms AS, et al. Genotype-dependent and -independent calcium signaling dysregulation in human hypertrophic cardiomyopathy. Circulation. 2016;134(22):1738-1748.

29. Glazier AA, et al. HSC70 is a chaperone for wild-type and mutant cardiac myosin binding protein C. JCI Insight. 2018;3(11):99319.

30. Lehman SJ, et al. Chronic calmodulin-kinase II activation drives disease progression in mutation-specific hypertrophic cardiomyopathy. Circulation. 2019;139(12):1517-1529.

31. Chopra A, et al. Force generation via $\beta$-cardiac myosin, titin, and $\alpha$-actinin drives cardiac sarcomere assembly from cell-matrix adhesions. Dev Cell. 2018;44(1):87-96.e5.

32. Coumans JV, et al. Variations in the relative mRNA levels of actins and myosin heavy chains do not produce corresponding differences in their proteins in the adult human heart. J Mol Cell Cardiol. 1997;29(3):895-905.

33. Predmore JM, et al. Ubiquitin proteasome dysfunction in human hypertrophic and dilated cardiomyopathies. Circulation. 2010;121(8):997-1004.

34. Schlossarek S, Frey N, Carrier L. Ubiquitin-proteasome system and hereditary cardiomyopathies. J Mol Cell Cardiol. 2014;71:25-31.

35. Dorsch LM, et al. Protein quality control activation and microtubule remodeling in hypertrophic cardiomyopathy. Cells. 2019;8(7):E741.

36. Coppini R, et al. Late sodium current inhibition reverses electromechanical dysfunction in human hypertrophic cardiomyopathy. Circulation. 2013;127(5):575-584

37. van Dijk SJ, et al. Contractile dysfunction irrespective of the mutant protein in human hypertrophic cardiomyopathy with normal systolic function. Circ Heart Fail. 2012;5(1):36-46.

38. Varnava AM, Elliott PM, Sharma S, McKenna WJ, Davies MJ. Hypertrophic cardiomyopathy: the interrelation of disarray, fibrosis, and small vessel disease. Heart. 2000;84(5):476-482.

39. Carrier L, et al. Asymmetric septal hypertrophy in heterozygous cMyBP-C null mice. Cardiovasc Res. 2004;63(2):293-304.

40. Toepfer CN, et al. Hypertrophic cardiomyopathy mutations in MYBPC3 dysregulate myosin. Sci Transl Med. 2019;11(476):eaat1199.

41. Chen PP, Patel JR, Powers PA, Fitzsimons DP, Moss RL. Dissociation of structural and functional phenotypes in cardiac myosin-binding protein C conditional knockout mice. Circulation. 2012;126(10):1194-1205.

42. Stöhr A, et al. Contractile abnormalities and altered drug response in engineered heart tissue from Mybpc3-targeted knock-in mice. J Mol Cell Cardiol. 2013;63:189-198.

43. Ma Z, et al. Contractile deficits in engineered cardiac microtissues as a result of MYBPC3 deficiency and mechanical overload. Nat Biomed Eng. 2018;2(12):955-967.

44. Yu J, et al. Human induced pluripotent stem cells free of vector and transgene sequences. Science. 2009;324(5928):797-801.

45. Hwang HS, et al. Comparable calcium handling of human iPSC-derived cardiomyocytes generated by multiple laboratories. J Mol Cell Cardiol. 2015;85:79-88.

46. Lian X, et al. Robust cardiomyocyte differentiation from human pluripotent stem cells via temporal modulation of canonical Wnt signaling. Proc Natl Acad Sci USA. 2012;109(27):E1848-E1857.

47. Herron TJ, et al. Extracellular matrix-mediated maturation of human pluripotent stem cell-derived cardiac monolayer structure and electrophysiological function. Circ Arrhythm Electrophysiol. 2016;9(4):e003638.

48. Tohyama S, et al. Distinct metabolic flow enables large-scale purification of mouse and human pluripotent stem cell-derived cardiomyocytes. Cell Stem Cell. 2013;12(1):127-137.

49. Burridge PW, Holmström A, Wu JC. Chemically defined culture and cardiomyocyte differentiation of human pluripotent stem cells. Curr Protoc Hum Genet. 2015;87:21.3.1-21.3.15.

50. Mali P, et al. RNA-guided human genome engineering via Cas9. Science. 2013;339(6121):823-826.

51. Ding Q, Regan SN, Xia Y, Oostrom LA, Cowan CA, Musunuru K. Enhanced efficiency of human pluripotent stem cell genome editing through replacing TALENs with CRISPRs. Cell Stem Cell. 2013;12(4):393-394.

52. Peters, DT, Cowan, CA, Musunuru, K. Genome editing in human pluripotent stem cells. StemBook. http://www.stembook. org/node/1438. Accessed January 6, 2020.

53. Grevesse T, Versaevel M, Circelli G, Desprez S, Gabriele S. A simple route to functionalize polyacrylamide hydrogels for the independent tuning of mechanotransduction cues. Lab Chip. 2013;13(5):777-780.

54. Dobin A, et al. STAR: ultrafast universal RNA-seq aligner. Bioinformatics. 2013;29(1):15-21.

55. Love MI, Huber W, Anders S. Moderated estimation of fold change and dispersion for RNA-seq data with DESeq2. Genome Biol. 2014;15(12):550.

56. Risso D, Ngai J, Speed TP, Dudoit S. Normalization of RNA-seq data using factor analysis of control genes or samples. Nat Biotechnol. 2014;32(9):896-902. 
57. Eisenberg E, Levanon EY. Human housekeeping genes, revisited. Trends Genet. 2013;29(10):569-574.

58. Ward JM, et al. Expression and methylation data from SLE patient and healthy control blood samples subdivided with respect to ARID3a levels. Data Brief. 2016;9:213-219.

59. Hellerstein MK, Neese RA. Mass isotopomer distribution analysis: a technique for measuring biosynthesis and turnover of polymers. Am J Physiol. 1992;263(5 Pt 1):E988-1001. 